\title{
FATORES QUE INFLUENCIAM NA INCIDÊNCIA DA DEPRESSÃO EM PACIENTES ONCOLÓGICOS E SUAS PRINCIPAIS CONSEQUÊNCIAS: UMA REVISÃO DE LITERATURA
}

Eveny Victória Siqueira de Nazaré ${ }^{1}$, Suzi Caroline Medeiros da Silva ${ }^{1}$, Marcella Kelly Costa de Almeida ${ }^{1}$. Carla de Castro Sant'Anna ${ }^{\mathbf{1 2}}$.

\author{
${ }^{1}$ Universidade da Amazônia \\ 2 Universidade Federal do Pará
}

E-mail para correspondência: santannacarla@yahoo.com.br

Submetido em: 26/11/2020 e aprovado em: 18/12/2020

\begin{abstract}
RESUMO
Introdução: A depressão é uma doença multifatorial caracterizada por interações psicológicas, orgânicas e ambientais, sendo frequentemente encontrada em pacientes oncológicos. Objetivo: Identificar os fatores de risco para o desencadeamento da depressão em pacientes oncológicos. Métodos: Foi realizada uma revisão de literatura incluídos todos os tipos de estudos que abordassem o tema publicados no período de 2009 a setembro de 2020, através de plataformas MEDLINE, SCIELO, LILACS, PUBMED, diretório Google Acadêmico. Resultados e discussão: Foram identificados 93 artigos de acordo com os descritores, no qual houve exclusão de 45, restando 48 para avaliação do título e resumo. Após esta análise, foi observado que 33 artigos não se enquadravam nos critérios de inclusão, onde somente 15 foram incluídos nesta revisão. A maioria dos estudos revelou que a maior prevalência de depressão foi encontrada na população feminina com idade entre 40 a 60 anos com câncer de mama, e a segunda maior taxa de transtornos depressivos foi observado em jovens de ambos os sexos na faixa etária de 15 a 19 anos. Conclusões: A maior progressão da depressão em pacientes oncológicos é observada em mulheres com câncer de mama e jovens de ambos os sexos acometidos com leucemia ou linfoma.

Palavras Chaves: Oncologia; Depressão; Câncer; Psicológico.
\end{abstract}

\begin{abstract}
Introduction: Depression is a multifactorial disease characterized by psychological, organic and environmental interactions and is frequently found in cancer patients. Aims: To identify the risk factors for triggering depression in cancer patients, age group, most affected gender, treatment
\end{abstract}


and the prevalence of depression in cancer patients. Methods: A literature review was carried out including all types of studies that addressed the topic published in the period 2009 to september 2020, through MEDLINE, SCIELO, LILACS, PUBMED, Google Scholar directory. Results and discussion: 93 articles were identified according to the descriptors, in which 45 were excluded, leaving 48 to assess the title and summary. After this analysis,t it was observed that 33 articles did not fit the inclusion criteria, where only 15 were included in this review. The highest prevalence of depression found was $5.9 \%$ in the female population without breast cancer, $21.6 \%$ had subsyndromic depressive symptoms, in women aged 40 to 60 years with breast cancer, and the second highest rate of depressive was observed in young men and women aged 15 to 19 years. Conclusions: The greatest progression of depression in cancer patients is in young women, due to the treatment together with the adverse reactions of chemotherapeutic agents. Having a high incidence of depression in cancer patients with a rate of $22 \%$ to $29 \%$ with variations, also showing a predominance of depression in women with breast cancer and in young men and women with leukemia and lymphoma.

Keywords: Oncology, Mental disorder; Cancer; Psychological.

\section{INTRODUÇÃO}

A depressão é classificada como um transtorno mental que envolve a interação entre fatores orgânicos, psicológicos e ambientais e seus principais sintomas são a angustia, rebaixamento de humor, perda de interesse, apatia, choro persistente, sentimento de impotência e perda de prazer pela vida. Considera-se como uma doença biológica em que pode ser tratada com medicamentos antidepressivos e intervenções psicossociais, como ativação comportamental e terapias $^{(1,2)}$.

A depressão é uma doença multifatorial, que apresenta uma grande variedade de fatores que podem desencadear o aparecimento desta. Pacientes com diagnóstico de câncer pode vir a desenvolver ansiedade e depressão, pois se deparam com uma doença agressiva e que altera o seu modo de viver e pensar. Além disso, indivíduos com câncer e outras condições médicas tem um risco maior para apresentar sintomas e transtornos depressivos persistentes. ${ }^{(3,4)}$

A partir do diagnóstico, alterações físicas e psicológicas provocadas pelo câncer, pode-se evidenciar a depressão, em que essas alterações persistem durante todo o tratamento e está relacionada com os efeitos colaterais do mesmo. Pacientes oncológicos tendem a ser mais 
suscetível a apresentar o quadro depressivo, porém, o diagnóstico pode ficar confuso, não sendo perceptivo pelos pacientes, pois os sintomas do câncer e efeitos colaterais do tratamento acabam se assemelhando aos sintomas da depressão e isso, consequentemente, pode acarretar na não adesão do tratamento do transtorno psiquiátrico. ${ }^{(5)}$

De acordo com a Organização Mundial de Saúde (OMS), o número de casos de depressão obteve um aumento de 18,4\% entre o período de 2005 a 2015, sendo 322 milhões de pessoas em todo o mundo, em que a maioria são mulheres (OMS, 2017). ${ }^{(6)}$ No Brasil, atinge 11,5 milhões de pessoas. A correlação da depressão com a piora de quadros clínicos, tendo em destaque: cardiopatias, diabetes, obesidade e problemas oncológicos. ${ }^{(7)}$

A taxa de prevalência da depressão é maior em pacientes oncológicos do que na população em geral, situa-se entre $22 \%$ e $29 \%$ e esta variação pode estar associada a sítios do tumor, estágio clínico, dor, funcionamento físico limitado, além da existência de suporte social. A associação da depressão com o câncer pode variar conforme a evolução da doença e dos tratamentos utilizados, sendo $14 \%$ dos pacientes ambulatórios, $28 \%$ dos pacientes em unidade de cuidados paliativos e $14,1 \%$ dos pacientes internados para transplante de medula óssea. ${ }^{(3)}$

De acordo com os estudos de Ferreira e colaboradores ${ }^{(4)}$, a prevalência da depressão em pacientes com câncer é de 26,19\% comparadas ao estudo de Bottino e colaboradores ${ }^{(3)}$ que encontrou uma prevalência de $22 \%$. Alguns fatores de risco podem contribuir para o desenvolvimento, como sexo feminino, tempo e tipo de tratamento, idade e tipo de câncer. Geralmente, a depressão é encontrada em estágios mais avançados do câncer, tendo uma predominância maior no sexo feminino. ${ }^{(8)}$

A depressão associada ao câncer também apresenta uma progressão maior em pacientes jovens do sexo feminino, por serem de fato mais vulneráveis a terem depressão, levando em consideração a questão hormonal que pode levar muitos a terem ansiedade e afetar um conjunto de fatores, como, seus sonhos, relacionamentos, família, trabalho, entre outros. ${ }^{(9)}$

A alta prevalência da depressão em pacientes com câncer pode estar correlacionada com a doença como também o tratamento, principalmente na quimioterapia. ${ }^{(10)}$ Além disso, a depressão pode ser um fator de gravidade e do número de eventos adversos da terapia quimioterápica, em que compromete o desenvolvimento de atividades cotidianas, do papel social e da adesão e continuidade do tratamento, provocando assim uma diminuição na qualidade de vida desses indivíduos. ${ }^{(8)}$ Desta forma, o objetivo do trabalho foi identificar os principais fatores que influenciam o desencadeamento da depressão em pacientes oncológicos. 


\section{MÉTODOS}

Foram realizados estudos cerca dos fatores e fenômenos que desencadeiam a depressão em pacientes oncológicos e suas principais consequências, utilizando artigos científicos publicados em periódicos nacionais.

\section{CRITÉRIOS DE ELEGIBILIDADE}

Foram incluídos estudos realizados no Brasil retrospectivos e prospectivos que abordassem fatores e fenômenos que desencadeiam a depressão em pacientes oncológicos e suas principais consequências, de todas as faixas etárias e tipos de câncer, publicados em inglês e português com texto completo de publicações em 2009 a 2020, além de site oficiais.

Trabalhos publicados em anais de congresso, artigos publicados em revistas não indexadas em banco de dados selecionados, em duplicatas foram excluídos da pesquisa.

\section{OBTENÇÃO DA AMOSTRA}

Os artigos usados neste trabalho foram publicados no período de 2009 a 2020, indexados nos bancos de dados Medical LiteratureAnalysisandRetrieval System Online (MEDLINE), ScientificElectronic Library Online (SCIELO), Literatura Latino-Americana e do Caribe em Ciências da Saúde (LILACS), US National Library of Medicine NationalInstitutesof Health (PUBMED), diretório Google Acadêmico e sites do Ministérios da Saúde. A estratégia de busca do PUBMED foi criada e adaptada a outros bancos de dados.

Os descritores específicos (em inglês e português) investigados foram: oncologia; depressão; câncer; transtorno emocional; fatores de risco; índice, vinculados aos operadores boleanos (AND e OR).

\section{SELEÇÃO DO ESTUDO}

Os autores da revisão trabalhando de forma independente, digitalizaram o resumo (abstract) e título de cada pesquisa. Todos os artigos que se enquadravam nos critérios de inclusão foram investigados por completo através de uma leitura minuciosa. Caso existisse diferenças entre as opiniões, um quarto autor foi solicitado para revisar e chegar a uma decisão final sobre a inclusão do estudo. Os autores realizaram a extração dos dados. 


\section{ANÁLISE DOS DADOS}

Utilizamos dados obtidos através de tabelas, gráficos e amostragem, cujo objetivo específico é analisar o sexo, faixa etária, tipos de câncer, diagnóstico e tratamentos organizados em uma plataforma do Excel (Microsoft Office 2017), sendo gerados os resultados a partir da análise de cada uma das variáveis.

\section{RESULTADOS E DISCUSSÃO}

A estratégia da pesquisa identificou 93 artigos. Desse total, foram excluídos 37 pela data de publicação, 4 pelo idioma diferente de português e inglês e 4 por texto incompleto ou duplicata e que não abordavam o tema, resultando em 48 artigos para avaliação do título do resumo. Nesta fase, foram excluídos 30 que não tratavam da questão de pesquisa, 15 estudos para leitura em texto selecionado completo e incluídos nesta revisão (Figura 1).

Figura 1. Fluxograma da pesquisa bibliográfica e seleção dos artigos.

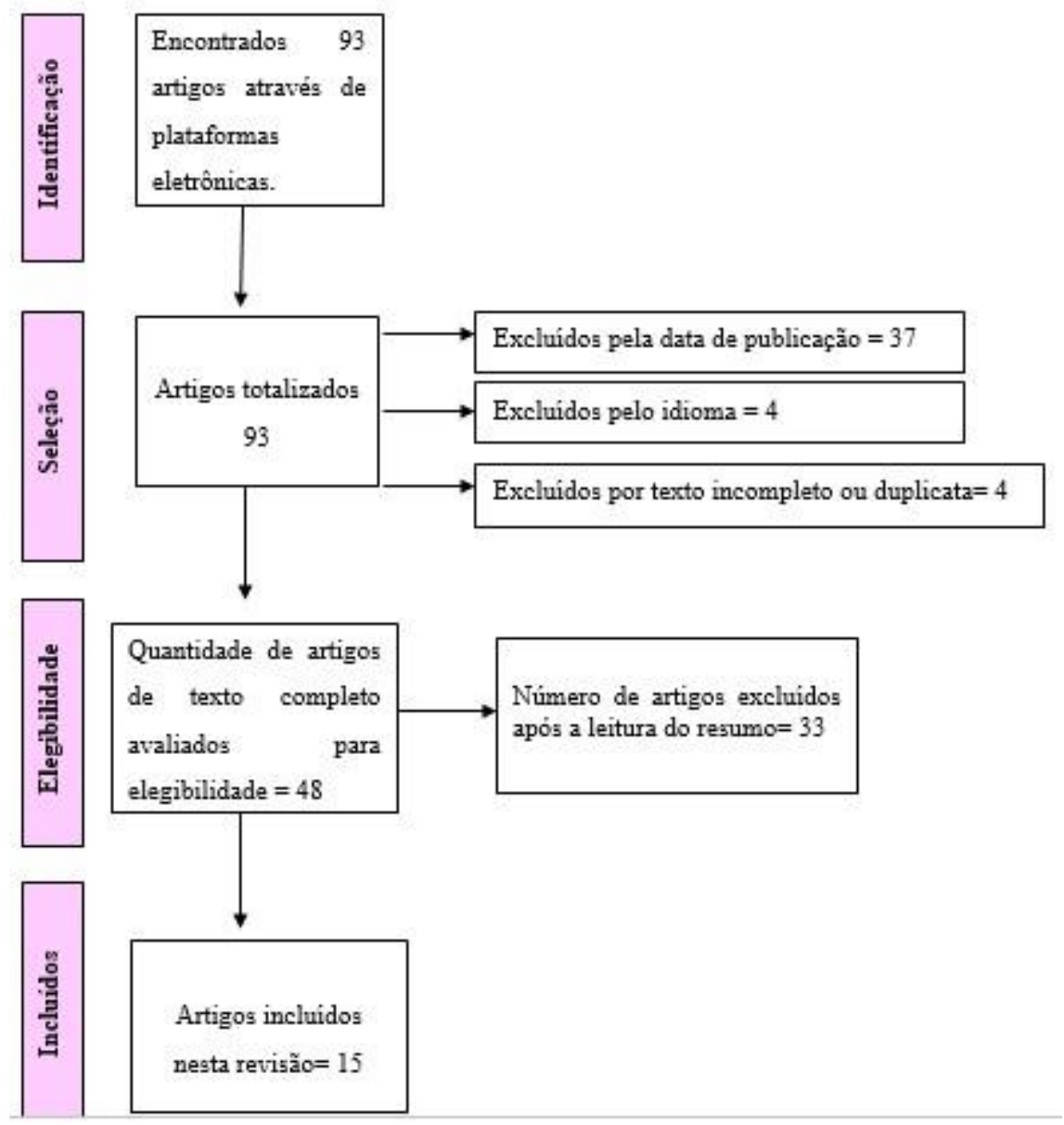

Fonte: Os autores (2020) 
Dos 15 estudos selecionados através de banco de dados, 5 foram no Periódicos, 5 no SCIELO, 1 na PUBMED, 1 em Repositório, 1 na revista RBC e 1 na NCBI, sendo 13 em português entre o período de 2009 a 2019 e 2 em idioma inglês no período de 2020. O quadro 1 apresenta a distribuição dos 15 artigos com estudos relevantes para pesquisa

Quadro 1 - Distribuição dos estudos incluídos segundo ano de publicação, autores, país de origem, título e revista.

\begin{tabular}{|c|c|c|c|c|c|}
\hline AUTORES & DATA & TÍTULO & OBJETIVOS & RESULTADOS & $\begin{array}{c}\text { BANCO DE } \\
\text { DADOS }\end{array}$ \\
\hline $\begin{array}{c}\text { Arantes, T.C } \\
\text { et al. }\end{array}$ & 2019 & $\begin{array}{l}\text { Fatores associados à } \\
\text { depressão em } \\
\text { pacientes } \\
\text { oncológicos durante } \\
\text { a quimioterapia. }\end{array}$ & $\begin{array}{l}\text { Analisar os fatores } \\
\text { associados à } \\
\text { depressão, em } \\
\text { pacientes } \\
\text { oncológicos, } \\
\text { durante } \\
\text { quimioterapia. }\end{array}$ & $\begin{array}{l}\text { Maior } \\
\text { prevalência em } \\
\text { mulheres entre } \\
40 \text { e } 79 \text { anos, } \\
\text { onde } 71,2 \% \text {, e } \\
17,3 \% \\
\text { apresentaram } \\
\text { depressão } \\
11,5 \% \text { disforria. } \\
\text { Pacientes que } \\
\text { iniciaram } \\
\text { quimioterapia } \\
\text { até os } 6 \text { meses } \\
\text { relatam mais } \\
\text { efeitos } \\
\text { colaterais. }\end{array}$ & $\begin{array}{l}\text { Revista Rene } \\
\text { UFC } \\
\text { (Universidade } \\
\text { Federal do } \\
\text { Ceará) }\end{array}$ \\
\hline $\begin{array}{c}\text { Araújo, R.V et } \\
\text { al }\end{array}$ & 2019 & $\begin{array}{l}\text { Efeito da meditação } \\
\text { no nível de estresse } \\
\text { psicológico de } \\
\text { mulheres com } \\
\text { neoplasia mamária: } \\
\text { revisão sistemática. }\end{array}$ & $\begin{array}{l}\text { Avaliar o efeito da } \\
\text { meditação no nível } \\
\text { de estresse } \\
\text { psicológico de } \\
\text { mulheres com } \\
\text { neoplasia mamária. }\end{array}$ & $\begin{array}{l}\text { Mulheres na } \\
\text { faixa etária entre } \\
46 \text { a } 63 \text { anos } \\
\text { considerando o } \\
\text { estágio do câncer } \\
\text { de mama, } \\
\text { tratamento, } \\
\text { diagnóstico de } \\
\text { outras doenças, } \\
\text { incluindo os } \\
\text { transtornos } \\
\text { mentais graves e }\end{array}$ & SCIELO \\
\hline
\end{tabular}




\begin{tabular}{|c|c|c|c|c|c|}
\hline & & & & $\begin{array}{l}\text { o uso de algumas } \\
\text { medicações. }\end{array}$ & \\
\hline $\begin{array}{c}\text { Assumpção, } \\
\text { G.LS }\end{array}$ & 2018 & $\begin{array}{l}\text { Depressão e suicídio: } \\
\text { Uma correlação }\end{array}$ & \begin{tabular}{lr}
\multicolumn{2}{l}{ Explanar e apontar } \\
sobre & as \\
influências & da \\
depressão & no \\
suicídio, & \\
descrevendo suas \\
principais \\
características, \\
com relatos sobre o \\
suicídio \\
decorrer \\
história.
\end{tabular} & $\begin{array}{l}\text { Constatar a } \\
\text { importância do } \\
\text { desenvolvimento } \\
\text { dos instrumentos } \\
\text { de prevenção e } \\
\text { qualificação dos } \\
\text { profissionais de } \\
\text { saúde. } \\
\text { Destacando os } \\
\text { métodos } \\
\text { utilizados na } \\
\text { psicoterapia para } \\
\text { o tratamento da } \\
\text { ideação suicida. }\end{array}$ & $\begin{array}{lr}\text { Revista } & \text { da } \\
\text { graduação } & \mathrm{em} \\
\text { Psicologia } & \text { PUC } \\
\text { MINAS } & \end{array}$ \\
\hline $\begin{array}{l}\text { Bottino, } \\
\text { S.M.B }\end{array}$ & 2009 & Depressão e câncer & $\begin{array}{l}\text { Avaliar a taxa de } \\
\text { prevalência } \\
\text { depressão } \\
\text { pacientes } \\
\text { oncológicos } \\
\text { internados } \\
\text { hospital no } \\
\text { Camargo A.C. } \\
\text { diferentes tipos de } \\
\text { câncer. }\end{array}$ & $\begin{array}{l}\text { Identificou a } \\
\text { vulnerabilidade } \\
\text { dos pacientes } \\
\text { com sintomas } \\
\text { depressivos e } \\
\text { ansiosos, } \\
\text { histórico de } \\
\text { depressão, dose e } \\
\text { duração } \\
\text { terapias, das } \\
\text { podendo ajudar o } \\
\text { médico no uso } \\
\text { criterioso dos } \\
\text { antidepressivos } \\
\text { em pacientes } \\
\text { com câncer. }\end{array}$ & SCIELO \\
\hline $\begin{array}{c}\text { Camurça, } \\
\text { R.T.S }\end{array}$ & 2016 & $\begin{array}{l}\text { Interferência dos } \\
\text { hormônios femininos } \\
\text { na ansiedade em } \\
\text { mulheres durante o } \\
\text { ciclo menstrual. }\end{array}$ & $\begin{array}{l}\text { Investigar a } \\
\text { ansiedade de } \\
\text { mulheres durante o } \\
\text { período menstrual. }\end{array}$ & $\begin{array}{l}\text { Foi observado } \\
\text { que não houve } \\
\text { padronização } \\
\text { dos instrumentos } \\
\text { para coleta de } \\
\text { dados e das fases }\end{array}$ & $\begin{array}{l}\text { Repositório } \\
\text { Institucional da } \\
\text { UFPB }\end{array}$ \\
\hline
\end{tabular}




\begin{tabular}{|c|c|c|c|c|c|}
\hline & & & & $\begin{array}{l}\text { do ciclo } \\
\text { menstrual. } \\
\text { Portanto, foram } \\
\text { relacionados os } \\
\text { níveis de } \\
\text { ansiedade com as } \\
\text { fases folicular e } \\
\text { lútea, sendo } \\
\text { encontrados } \\
\text { maiores } \\
\text { resultados na } \\
\text { fase lútea. }\end{array}$ & \\
\hline $\begin{array}{l}\text { Carvalho, } \\
\text { S.M.F }\end{array}$ & 2015 & $\begin{array}{l}\text { Prevalência maior } \\
\text { em pacientes com } \\
\text { câncer de mama. }\end{array}$ & $\begin{array}{l}\text { Determinar } a \\
\text { prevalência } \text { da } \\
\text { depressão maior } \\
\text { em mulheres com } \\
\text { câncer de mama. }\end{array}$ & $\begin{array}{l}\text { A maior } \\
\text { prevalência da } \\
\text { depressão } \\
\text { encontrada foi de } \\
5,9 \% \quad \text { em } \\
\text { mulheres com } \\
\text { câncer de mama, } \\
\text { observou-se na } \\
\text { população } \\
\text { feminina não } \\
\text { portadora de } \\
\text { câncer de mama } \\
21,6 \% \text { sintomas } \\
\text { depressivos. }\end{array}$ & $\begin{array}{l}\text { Periódicos } \\
\text { Eletrônicos em } \\
\text { Psicologia }\end{array}$ \\
\hline $\begin{array}{c}\text { Coutinho, } \\
\text { M.P.L }\end{array}$ & 2016 & $\begin{array}{l}\text { Relação entre } \\
\text { depressão } \\
\text { qualidade de vida de } \\
\text { adolescentes no } \\
\text { contexto escolar }\end{array}$ & $\begin{array}{l}\text { Verificar a relação } \\
\text { entre depressão e } \\
\text { qualidade de vida } \\
\text { de adolescentes no } \\
\text { contexto escolar. }\end{array}$ & $\begin{array}{l}\text { Foram } \\
\text { demonstrados } \\
\text { que } 8,3 \% \text { dos } \\
\text { adolescentes } \\
\text { tinham } \\
\text { indicativos de } \\
\text { sintomatologia } \\
\text { depressiva, } \\
\text { sendo que as } \\
\text { correlações mais } \\
\text { fortes foram os } \\
\text { fatores: estado }\end{array}$ & SCIELO \\
\hline
\end{tabular}




\begin{tabular}{|c|c|c|c|c|c|}
\hline & & & & $\begin{array}{l}\text { emocional } \quad(\mathrm{r}= \\
0,54), \\
\text { sentimentos } \\
(\mathrm{r}=0,65) \quad \mathrm{e} \\
\text { ambiente } \\
\text { familiar } \quad(\mathrm{r}= \\
0,54) .\end{array}$ & \\
\hline Couto, A.M.L & 2014 & $\begin{array}{l}\text { Sintomas } \\
\text { psiquiátricos em } \\
\text { pacientes em uso de } \\
\text { antitumorais. }\end{array}$ & $\begin{array}{l}\text { Verificar } \\
\text { incidência } \\
\text { características dos } \\
\text { efeitos } \\
\text { psiquiátricos } \\
\text { causados pelo uso } \\
\text { prolongado de } \\
\text { antitumorais } \\
\text { podendo favorecer } \\
\text { o aparecimento de } \\
\text { sintomas tais como } \\
\text { alterações } \\
\text { humor, ansiedade, } \\
\text { alucinações em } \\
\text { pacientes } \\
\text { oncológicos. }\end{array}$ & $\begin{array}{l}\text { O estudo busca } \\
\text { verificar se há } \\
\text { relação entre o } \\
\text { aparecimento de } \\
\text { efeitos } \\
\text { psiquiátricos, a } \\
\text { dosagem e o } \\
\text { tempo de } \\
\text { tratamento com } \\
\text { IFN alfa e o } \\
\text { indice de MAO } \\
\text { plaquetas no } \\
\text { sangue. } \\
\text { autores citam } \\
\text { que a disfunção } \\
\text { serotonérgica é } \\
\text { provavelmente } \\
\text { um dos } \\
\text { mecanismos } \\
\text { psicopatológicos } \\
\text { dos efeitos do } \\
\text { tratamento. }\end{array}$ & $\begin{array}{l}\text { Repositório } \\
\text { Institucional } \\
\text { UFBA }\end{array}$ \\
\hline Ferreira, A.S & 2016 & $\begin{array}{lr}\text { Prevalência } & \text { de } \\
\text { ansiedade } & \mathrm{e} \\
\text { depressão } & \mathrm{em} \\
\text { pacientes } & \\
\text { oncológicos } & \mathrm{e} \\
\text { identificação } & \mathrm{de} \\
\text { variáveis } & \\
\text { predisponentes } & \end{array}$ & $\begin{array}{lr}\text { Avaliar } & \text { a } \\
\text { ocorrência } & \text { de } \\
\text { depressão } & \text { e } \\
\text { ansiedade } & \text { em } \\
\text { pacientes } & \\
\text { oncológicos, além } \\
\text { de analisar as } \\
\text { associações entre } \\
\text { as variáveis }\end{array}$ & $\begin{array}{lr}\text { Foram } & \\
\text { encontrados } & \\
31,33 \% & \text { dos } \\
\text { pacientes } & \text { com } \\
\text { ansiedade } & \\
\text { provável } & \text { ou } \\
\text { possível, } & \text { e } \\
26,18 \% & \text { com } \\
\text { depressão } & \end{array}$ & $\begin{array}{l}\text { Revista } \\
\text { Brasileira de } \\
\text { Cancerologia }\end{array}$ \\
\hline
\end{tabular}




\begin{tabular}{|c|c|c|c|c|c|}
\hline & & & $\begin{array}{l}\text { clínicas } \\
\text { sociodemograficas, } \\
\text { e as comorbidades } \\
\text { psiquiátricas }\end{array}$ & $\begin{array}{l}\text { provável ou } \\
\text { possível. Após } \\
\text { correlação dos } \\
\text { dados } \\
\text { encontrados, não } \\
\text { se identificou } \\
\text { diferença nos } \\
\text { subgrupos, } \\
\text { porém houve } \\
\text { uma tendência } \\
\text { maior a mulheres } \\
\text { apresentarem } \\
\text { depressão. }\end{array}$ & \\
\hline Gabsi, A & 2020 & $\begin{array}{l}\text { Ansiedade } \\
\text { depressão em } \\
\text { pacientes com câncer } \\
\text { durante a pandemia } \\
\text { de COVID-19: um } \\
\text { estudo de centro } \\
\text { único }\end{array}$ & 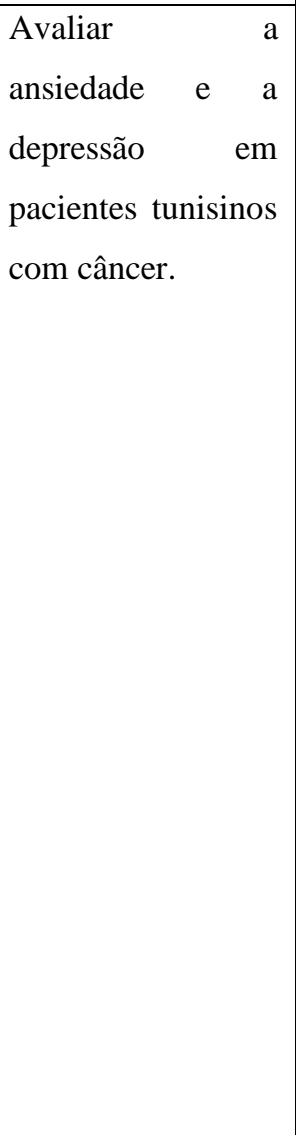 & $\begin{array}{l}\text { A incidência de } \\
\text { depressão sob } \\
\text { nova pandemia } \\
\text { de corona vírus } \\
\text { em pacientes } \\
\text { com câncer foi } \\
\text { de } \quad 29,7 \% \\
\text { (27/91). } \\
\text { Enquanto } \\
\text { incidência de } \\
\text { ansiedade foi de } \\
69,2 \% \text { (63/91). } \\
\text { Não houve } \\
\text { relações } \\
\text { significativas } \\
\text { entre depressão e } \\
\text { sexo, apoio } \\
\text { familiar, tipo de } \\
\text { câncer ou dor. }\end{array}$ & $\begin{array}{l}\text { NCBI } \\
\text { National Center } \\
\text { for } \\
\text { Biotetchnology } \\
\text { Information }\end{array}$ \\
\hline $\begin{array}{c}\text { Gonçalves, } \\
\text { A.M.C }\end{array}$ & 2018 & $\begin{array}{l}\text { Prevalência da } \\
\text { depressão e fatores } \\
\text { associados em } \\
\text { mulheres atendidas } \\
\text { pela estratégia de } \\
\text { saúde da família }\end{array}$ & $\begin{array}{l}\text { Avaliar a } \\
\text { prevalência de } \\
\text { depressão e os } \\
\text { fatores associados } \\
\text { em mulheres de } 20 \\
\text { a } 59 \text { anos de áreas }\end{array}$ & $\begin{array}{l}\text { Observou-se que } \\
1.958 \text { mulheres } \\
\text { incluídas nesta } \\
\text { análise, } 28,5 \% \\
\text { encontra-se na } \\
\text { faixa etária entre }\end{array}$ & SCIELO \\
\hline
\end{tabular}




\begin{tabular}{|c|c|c|c|c|c|}
\hline & & & $\begin{array}{l}\text { cobertas } \\
\text { Estratégia } \\
\text { Saúde da } \\
\text { de município da } \\
\text { Zona da Mata } \\
\text { Mineira }\end{array}$ & $\begin{array}{l}30 \text { e 39anos; } \\
\text { Fatores } \\
\text { associados a } \\
\text { depressão no } \\
\text { estudo realizado: } \\
\text { Baixa } \\
\text { escolaridade, não } \\
\text { possui trabalho, } \\
\text { sem apoio } \\
\text { familiar }\end{array}$ & \\
\hline $\begin{array}{c}\text { Nascimento, } \\
\text { T.R.S }\end{array}$ & 2018 & $\begin{array}{l}\text { O impacto } \\
\text { psicológico causado } \\
\text { pelo diagnóstico de } \\
\text { câncer } \\
\text { adolescentes }\end{array}$ & $\begin{array}{l}\text { O estudo visa } \\
\text { compreender } \\
\text { impacto } \\
\text { psicológico } \\
\text { causado } \\
\text { adolescente pelo } \\
\text { diagnóstico do } \\
\text { câncer } \\
\text { importância de um } \\
\text { psicólogo tendo em } \\
\text { vista o paciente e } \\
\text { sua família. }\end{array}$ & $\begin{array}{l}\text { Por meio deste } \\
\text { artigo entende-se } \\
\text { que } \\
\text { diagnóstico do } \\
\text { câncer na } \\
\text { adolescência } \\
\text { provoca } \\
\text { alterações na } \\
\text { vida do deles e } \\
\text { das pessoas } \\
\text { envolvidas. } \\
\text { Sentimentos } \\
\text { ruins podem } \\
\text { surgir, desde } \\
\text { ansiedade, medo, } \\
\text { raiva podendo } \\
\text { levar a estados } \\
\text { depressivos. }\end{array}$ & $\begin{array}{ll}\text { Repositório } & \text { do } \\
\text { Colégio } & \text { de } \\
\text { Psicologia } & \end{array}$ \\
\hline $\begin{array}{c}\text { Ravindran, } \\
\text { A.V }\end{array}$ & 2020 & $\begin{array}{l}\text { Depressão e } \\
\text { ansiedade em relação } \\
\text { à incidência e } \\
\text { mortalidade por } \\
\text { câncer: uma revisão } \\
\text { sistemática e meta- } \\
\text { análise de estudos de } \\
\text { coorte }\end{array}$ & $\begin{array}{lr}\text { Quantificar } & \text { a } \\
\text { associação entre } \\
\text { depressão } \\
\text { ansiedade definida } \\
\text { por escalas de } \\
\text { sintomas } \\
\text { diagnóstico clínico } \\
\text { e o risco de } \\
\text { incidência } \\
\text { câncer, de }\end{array}$ & $\begin{array}{l}\text { As análises } \\
\text { sugerem que a } \\
\text { depressão e a } \\
\text { ansiedade podem } \\
\text { ter um papel } \\
\text { etiológico e } \\
\text { impacto } \\
\text { prognóstico } \\
\text { sobre o câncer, } \\
\text { embora haja }\end{array}$ & PUBMED \\
\hline
\end{tabular}




\begin{tabular}{|c|c|c|c|c|c|}
\hline & & & $\begin{array}{l}\text { mortalidade por } \\
\text { todas as causas em } \\
\text { pacientes } \\
\text { oncológicos }\end{array}$ & $\begin{array}{l}\text { potencial } \\
\text { causalidade } \\
\text { reversa. }\end{array}$ & \\
\hline Souza, B.F & 2013 & $\begin{array}{l}\text { Pacientes em uso de } \\
\text { quimioterápicos: } \\
\text { Depressão e adesão } \\
\text { ao tratamento }\end{array}$ & $\begin{array}{l}\text { Verificar a adesão } \\
\text { ao tratamento } \\
\text { quimioterápico e } \\
\text { os sinais } \\
\text { indicativos de } \\
\text { depressão. }\end{array}$ & $\begin{array}{l}\text { Os resultados } \\
\text { revelaram que } \\
10,8 \% \text { e } 1,9 \% \\
\text { apresentam } \\
\text { depressão } \\
\text { moderada } \\
\text { grave. } \\
\text { Identificou-se } \\
\text { falta de adesão } \\
\text { ao tratamento em } \\
\text { 48\% dos } \\
\text { pacientes } \\
\text { associação } \\
\text { significativa } \\
\text { entre a presença } \\
\text { de depressão e as } \\
\text { variáveis renda } \\
\text { per capita, tempo } \\
\text { e quantidades de } \\
\text { cirurgias. }\end{array}$ & SCIELO \\
\hline Veneti, A & 2018 & $\begin{array}{l}\text { Avaliação } \\
\text { depressão } \\
\text { ansiedade } \\
\text { pacientes com câncer } \\
\text { de mama: } \\
\text { Prevalência e fatores } \\
\text { associados }\end{array}$ & $\begin{array}{l}\text { Avaliar } \\
\text { prevalência e os } \\
\text { fatores associados } \\
\text { de depressão e } \\
\text { ansiedade em } \\
\text { pacientes com } \\
\text { câncer de mama, a } \\
\text { fim de identificar } \\
\text { preditores } \\
\text { independentes de } \\
\text { risco } \\
\text { transtornos } \\
\text { mentais. }\end{array}$ & $\begin{array}{l}\text { Grande } \\
\text { percentual foi } \\
\text { classificado } \\
\text { como deprimido } \\
(38,2 \%) \\
\text { ansioso (32,2\%) } \\
\text { e os fatores } \\
\text { associados foram } \\
\text { idade, estado } \\
\text { civil, } \\
\text { escolaridade, } \\
\text { estágio do câncer } \\
\text { a partir de } \\
\text { análises }\end{array}$ & PUBMED \\
\hline
\end{tabular}




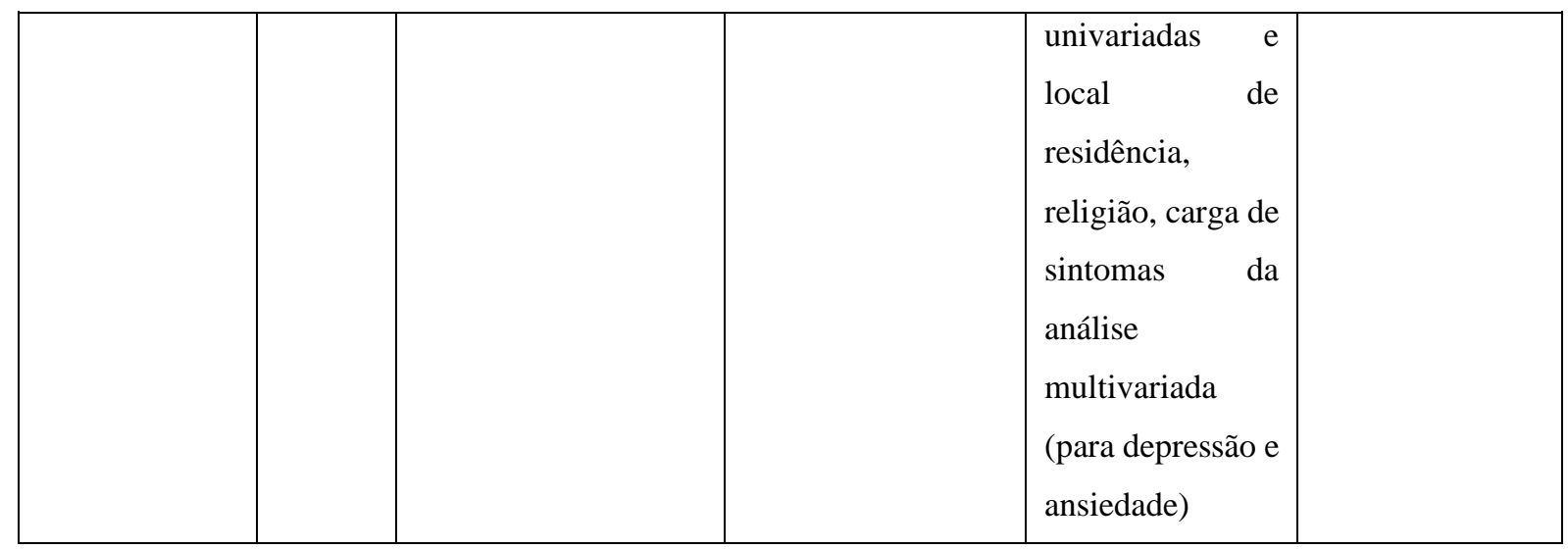

Fonte: Os autores (2020)

Os estudos encontrados mostram os fatores que influenciam a incidência da depressão em pacientes oncológicos no Brasil e suas principais consequências, no qual foi observado uma alta taxa de morbidade entre este grupo.

De acordo com o estudo de Arantes e colaboradores ${ }^{(9)}$, é possível observar que o tratamento com quimioterápico em mulheres com câncer de mama podem causar manifestações ou sintomas de depressão e ansiedade nesses pacientes, considerando alguns fatores, como idade, sexo, tipo de câncer, diagnostico, escolaridade, outras terapias e tempo de tratamento podendo influenciar na intensidade dos sintomas.

O câncer de mama se destaca pela maior quantidade de mortes entre mulheres no Brasil e no mundo, levando em consideração as mudanças físicas e psicológicas, sendo impactantes desde o momento que o diagnóstico é anunciado, esses pacientes deparam-se com uma doença angustiante e risco de morte, com receio da mesma ser incurável podendo ser afetado uma parte do corpo ocasionando em sofrimento e dor. Ainda que sintomas depressivos sejam muito comuns em pacientes oncológicos, às vezes, acabam passando despercebidos ou não tratados corretamente quando se obtém um diagnóstico, pois estão associados depressão a pior prognóstico e alta taxa de mortalidade por câncer, apresentando menor adesão ao tratamento por conta de sintomas depressivos. Além disso, alguns fatores como, efeitos adversos da medicação, mudanças comportamentais, deficiência na comunicação entre pacientes e profissionais da saúde, suporte social insuficiente e crenças, portanto é de suma importância a verificação desses sintomas depressivos e da adesão ao tratamento para a melhora da qualidade de vida. $^{(11)}$ 
O uso de terapias antineoplásicas como interferon, vimblastina, vincristina, asparaginase, além de outros quimioterápicos e corticoides levam a quadro depressivos. ${ }^{(5)} \mathrm{O}$ estudo de Nascimento ${ }^{(12)}$ mostrou que o tempo de diagnóstico e tratamento, são importantes variáveis associadas a prevalência de depressão em pacientes oncológicos, havendo possibilidade de ineficácia ou de não adesão ao tratamento. Entretanto a literatura revela que os antidepressivos são efetivos e proporcionam melhor adesão aos tratamentos de câncer e que o tratamento prévio com esses medicamentos pode minimizar sintomas de depressão em pacientes oncológicos.

Segundo Carvalho e colaboradores ${ }^{(13)}$ a prevalência de depressão maior encontrada foi de 5,9\% semelhante a observada na população feminina não portadora de câncer de mama, 21,6\% apresentam sintomas depressivos subsindrômicos. Assim, concluiu-se neste estudo alta taxa de depressão e ansiedade sendo mais vulneráveis no sexo feminino com idade de 40 a 60 anos onde o câncer de mama possui uma elevada incidência no Brasil, e a segunda taxa mais elevada de transtornos depressivos está entre os jovens e adolescentes de ambos os sexos na faixa etária de 15 a 19 anos.

Uma pesquisa feita nos Estados Unidos aponta que cerca de 70.000 mil pessoas são diagnosticadas com câncer e milhares de indivíduos concluem o tratamento anualmente. Indivíduos mais jovens desencadeiam desproporcionalmente certos tipos de câncer, principalmente linfomas e leucemias, câncer de pele, câncer endócrino, tumores no cérebro, na medula espinhal e câncer de mama. A taxa de transtorno mental, depressão ou ansiedade variou de $16 \%$ a $42 \%$, estima-se $20 \%$ de depressão leve em pacientes oncológicos, tendo a fadiga como efeito adverso mais comum em terapia contra o câncer, alguns sintomas físicos e afetivos está o fardo de viver com ameaça existencial de uma vida encurtada, podendo está relacionado ao diagnostico para transtorno depressivo mental, dificultando nas amostras clínicas. Para esses pacientes o medo do avanço ou recorrência do câncer pode influenciar consideravelmente em todos os aspectos de sua vida. ${ }^{(14)}$

A literatura evidencia informações de que a depressão pode ser duas vezes maior em mulheres do que em homens, considerando fatores fisiológicos e hormonais. Além disso, nos últimos anos, a mulher vem contribuindo diretamente no sustento da família, com as responsabilidades do lar, maternidade, dentre outros, que podem acarretar na sobrecarga de trabalho, sendo um fator de risco para a saúde. ${ }^{(7)}$

Apesar de determinadas pessoas serem propensas geneticamente para o acréscimo de alguns tipos de tumor, o surgimento da patologia envolve, fatores comportamentais, de estilo 
de vida, do mesmo modo que os tumores afetam as mamas, constituindo-se como um dos mais comuns entre as mulheres brasileiras, tendo um grande impacto na sua feminilidade e sexualidade. Contudo, alguns fatores de risco envolvem envelhecimento, pré-disposição genética, ingestão de álcool, obesidade, exposição a radiação ionizante, uso prolongado de contraceptivos orais juntamente a cirurgia e radioterapia como formas de tratamento local. $\mathrm{O}$ procedimento cirúrgico pode ocorrer de maneira onde será necessário a retirada da parte da glândula mamária que contém o tumor, ou a mastectomia retirando a glândula mamária total. ${ }^{(15)}$

No Brasil o diagnóstico tem no estágio avançado (III e VI) da doença, dificultando o tratamento, elevando a quantidade de mastectomias realizadas no país e consequentemente haverá perda na qualidade de vida desses pacientes. Além disso, o tratamento pode causar a perda dos cabelos, a irregularidade na menstruação, perda de infertibilidade, afetando ainda mais a identidade da mulher, levando o sentimento de baixa autoestima, inferioridade e medo. ${ }^{(16)}$

\section{CONCLUSÃO}

Este estudo identificou os fatores de risco para o desencadeamento da depressão em pacientes oncológicos, como o tipo de câncer, tempo de tratamento, sítio do tumor, estágio clínico, dor, funcionamento físico limitado, existência do suporte social, evolução da doença e efeitos colaterais da terapia quimioterápica.

A alta prevalência da depressão, em pacientes com câncer pode estar relacionada com a doença, como também pelo tratamento escolhido, o que pode comprometer a adesão ao tratamento, provocando uma diminuição na qualidade de vida deste paciente.

A maior progressão da depressão em pacientes com câncer é em jovens do sexo feminino, levando em consideração questões hormonais, relacionamento, trabalho, família, sonhos, entre outros. Sendo este a segunda maior causa de morte no Brasil neste grupo.

O uso de terapias antineoplasicas como Interferon, vimblastina, vincristina, asparaginase, entre outros quimioterápicos e corticoides, levam a quadro depressivo havendo a possibilidade a não adesão ao tratamento. Concluiu-se nesse estudo as imprecisas taxas de todos os transtornos de humor que são substancialmente mais altas em pacientes com câncer do que na população em geral. 


\section{REFERÊNCIAS BIBLIOGRÁFICAS}

1. Assumpção GLS, Oliveira LA, Souza MFS. Depressão e suicídio: uma correlação. Revista da Graduação em Psicologia da Pontifícia Universidade Católica de Minas.2018, 3(5); 312333.

2. Brasil. Ministério da Saúde. Disponível em: https://bvsms.saude.gov.br/. Acesso em: 18 fev. 2020.

3. Bottino SMB, Fráguas R, Gattaz WF. Depressão e câncer. Revista de Psiquiatria Clínica. 2009, 36(3); 109-15.

4. Ferreira AS, Bicalho BP, Neves LFG, Menezes MT, Silva TA, Faier TA, Machado RM. Prevalência de ansiedade e depressão em pacientes oncológicos e identificação de variáveis predisponentes. Revista Brasileira de Cancerologia. 2016, 62(4); 321-228.

5. Souza BF, Pires FH, Dewulf NLS, Inocenti A, Silva AEB, Miasso AI. Pacientes em uso de quimioterápicos: depressão e adesão ao tratamento. Revista da Escola de Enfermagem da Universidade de São Paulo. 2013, 47(1); 61-8.

6. World Health Organization (WHO). Depression and Other Common Mental DisordersGlobal Health $\quad$ Estimates. 2017.2 Disponível em: http://apps.who.int/iris/bitstream/10665/254610/1/WHO-MSD-MER-2017.2eng.pdf?ua=1.

7.Gonçalves AMC, Gamarra CJ, Lopes CS, Silva GA, Gama JRA, Duque KCD, Machado MLSM, Teixeira MTB. Prevalência de depressão e fatores associados em mulheres atendidas pela Estratégia de Saúde da Família. Jornal Brasileiro de Psicologia. 2018, 67(2); 101-9.

8.Castro EK, Romeiro FB, Lima NB, Lawrenz P, Hass S. Percepção da doença, indicadores de ansiedade e depressão em mulheres com câncer. Psicologia, Saúde \& Doenças. 2015, 16(3).

9. Camurça RTS. Interferência dos hormônios femininos na ansiedade em mulheres durante o ciclo menstrual. Dissertação de Mestrado (Pós-Graduação em Neurociência cognitiva e comportamento). Centro de Ciências Humanas Letras e Artes, Universidade Federal da Paraíba, João Pessoa. 2016, 1-65.

10. Arantes TC, Martins VE, Mendes AS, Silva AMB, Nicolussi AC. Fatores associados à depressão em pacientes oncológicos durante quimioterapia. Revista Rene. 2019, 20; 1-8.

11. Silva LC. Câncer de mama e sofrimento psicológico: aspectos relacionados ao feminino. Psicologia em estudo Maringá. 2008, 13(2).

12. Miasso AI, Inocenti A, Silva AEB, Souza BF, Moraes JA, Santos MA. Mulheres com câncer de mama em quimioterapia: sintomas de depressão e adesão ao tratamento. Revista LatinoAmericana de enfermagem. 2014, 22(5). 
13. Nascimento TRS, Loiola KS. O Impacto psicológico causado pelo diagnóstico de câncer em adolescentes. Colegiado de Psicologia Unime. 2018. Disponível em: https://repositorio.pgsskroton.com.br/bitstream/123456789/23636/1/O\%20IMPACTO\%20PSI COL $\%$ C3\%93GICO $\% 20$ CAUSADO $\% 20$ PELO $\% 20$ DIAGN\%C3\%93STICO $\% 20 \mathrm{DE} \% 20 \mathrm{C} \%$ C3\%82NCER\%20EM\%20ADOLESCENTES\%20Thatyhellen\%20Nascimento\%20e\%20Kar en\%20Loiola.pdf. Acesso em: 05 mar. 2020.

14. Carvalho SMF, Bezerra IMP, Freitas TH, Rodrigues RCS, Carvalho IOC, Brasil AQ, Júnior FTC, Diniz LFB, Paz-Cox A, Abreu LC. Prevalência de depressão maior em pacientes com câncer de mama.JournalofHumanGrowthandDevelopment. 2015, 25(1); 68-74.

15. Rosenstein DL, Park EM. Depression in adolescentsandyoungadultswhit câncer. Dialogues ClinNeurosci. 2015, 17(2); 171-180.

16. Araujo RV, Fernandes AFC, Nery IS, Andrade EMLR, Nogueira LT, AZEVEDO, F.H.C. Efeito da meditação no nível de estresse psicológico de mulheres com neoplasia mamária: revisão sistemática. Revista da Escola de Enfermagem da Universidade de São Paulo. 2019, $53 ; 1-10$.

17. Ravidran AV, Leung J, Liu JJ, Que JY, Qiao JL, Shi J, Shi JF, Lappin JM, Lu L, Qiao VL, Chen WQ, Bao YP, Wang YH. Depression and anxiety in relation to cancer incidence and mortality: a systematic review and meta-analysis of cohort studies. Psiquiatria Molecular.2020, 25 (7); 1487-1499.

18. Coutinho MPL, Pinto AVL, Cavalcanti JG, Araújo LS, Coutinho ML. Relação entre depressão e qualidade de vida de adolescentes no contexto escolar. Psicologia, saúde \& Doença. 2016, 17(3); 338-351.

19. Couto AML. Sintomas psiquiátricos em pacientes em uso de antitumorais. Trabalho de Conclusão de Curso (Graduação em Medicina). Faculdade de Medicina, Universidade Federal da Bahia, Salvador. 2014, 1-26.

20. Veneti A, Mitsi D, Fradelos EC, Papathanasiou IV, Tsaras H, Kelesi M, Zyga S. Assessmentofdepressionandanxiety in patientswithbreastcancer: prevalenceandassociatedfactors. Jornal acadêmico de oncologia. 2018, 19 (6); 1661-1669.

21. Gabsi A, Mezlini A, Mokrani A, Letaief-Ksontini F, Mghirbi F, Rais H, Ayadi M, Chraiet N; Yahyaoui Y, Zenzri Y. 1573P Anxiety and depression in cancer patients during the COVID19 pandemic: a single center study. Biblioteca Nacional de Medicina dos EUA. 2020, 31; S957 $-\mathrm{S} 958$. 